\title{
Catapult Description of Magnetic Fields and Forces
}

\author{
Martin McCall ${ }^{1}$, Paul Kinsler ${ }^{2,3,1}$, and Vijay Tymms ${ }^{1}$ \\ 1 Department of Physics, Imperial College London, United Kingdom SW7 $2 A Z$ \\ ${ }^{2}$ Cockcroft Institute, Sci-Tech Daresbury, Daresbury WA4 4AD, UK and \\ 3 Physics Department, Lancaster University, Lancaster LA1 4YB, UK
}

\begin{abstract}
The 'catapult' picture for the forces on current-carrying wires in magnetic fields is a popular teaching aid, but its accuracy and utility are seriously questionable.
\end{abstract}

In many high-school teaching materials the force on a current-carrying wire in a magnetic field is discussed using an idea based on a so-called 'catapult field' (cf. Fig. 1); an engaging depiction of magnetic forces, albeit one which, to the best of our knowledge, has neither been used in college-level educational material, or scrutinized in academic journals. Here we examine carefully the physical basis of the 'catapult field' concept.

The catapult field picture is described in Fig. 1: if the background magnetic field is superposed with that from the current in the wire, the resultant field pattern can be pictured as a kind of catapult that pushes the wire in the correct direction, as indicated in the figure.
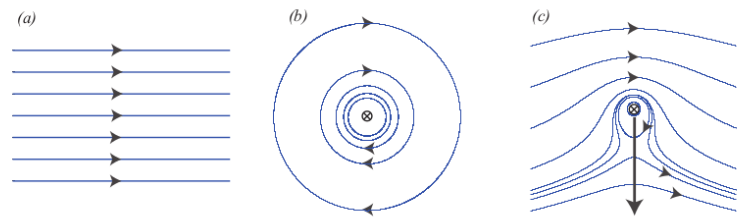

FIG. 1. The catapult effect: the summation of a uniform background magnetic field (a) with the concentric magnetic field of a current-carrying wire (b) gives a resultant 'catapult' field (c) which pushes the wire downwards.

A representative sample of commentary taken from educational material using the 'catapult' construction is:

1. The wire moves because the magnetic field of the permanent magnet reacts with magnetic field of the current in the wire...The wire tends to be 'catapulted' outwards.[1]

2. Why does the [wire] move? The magnetic field of the permanent magnets interacts with the magnetic field of the current in the [wire]. ... The diagram shows the combined field of the magnet and the [wire]: The lines of flux behave a bit like elastic bands. Can you see that the wire tends to be catapulted [downwards]?[2]

3. The wire moves away from the area of highest field intensity (where the magnetic field lines are closest) to a region [of] lower intensity. [3]

4. When the two fields are combined, ... the wire, if free to move, will be catapulted from the stronger field towards the weaker field... [4]

5 . The conductor moves from the region of greater flux density to smaller flux density. So [the wire] moves downwards as shown.[5]
Such descriptions, appearing along with diagrams similar to Fig. 1, seem to give at once a memorable and intuitive impression of how the force on the wire arises. However, the force on the wire is given by the Lorentz force law which tells us that the force is proportional to $\mathbf{I} \times \mathbf{B}$, where $\mathbf{B}$ is the background magnetic field at the wire. The Lorentz force law has no dependence on the magnetic field contributed by the current in the wire.

It might still be hoped that there is some description of the catapult picture which is nevertheless physically correct. Two related possibilities are suggested by the quotes given above: (a) that the wire is somehow pushed towards lower total magnetic field strengths from higher ones; and (b) the field lines are somehow 'elastic' and push on the wire as they try to straighten out. Both of these are compatible with a suggestion that the force is related to a gradient in the magnetic energy density. However, since magnetic forces do no work, the magnetic energy distribution does not change. Indeed, as can be seen from Fig. 1, the resultant field pattern would just move with the wire, so that there is no 'relaxation' of field lines to minimize the magnetic energy.

In lieu of any support from physical reasoning, it might nevertheless be argued that the catapult construction is simply a convenient reminder of which way the force on a wire acts; such as Fleming's left-hand rule. However, the left-hand rule is more than simply a reminder. The magnetic field itself can be introduced to describe the observation that a charged particle deviates perpendicular to its velocity and the direction of presumed field lines running between two magnetic poles. Once the direction of the field lines is fixed by convention, the direction of deviation is described by the left-hand rule, (i.e. Forefinger $\equiv$ Field, seCond finger $\equiv$ Current, thuMb $\equiv$ Force/motion). This embodies both our empirical observation, and what is later refined in our undergraduate physics education to be the Lorentz force law, a basic law of electrodynamics. By contrast, the catapult picture does not lead naturally to the Lorentz force law, and is not closely linked to a valid physical explanation.

Moreover, it is possible to construct a counter-example where the catapult picture is in direct conflict with the prediction of the Lorentz force law. Fig. 2(a) shows concentric conductors carrying opposing currents of magnitudes $I_{1}$ and $I_{2}$. The inner conductor is fixed, while the outer conductor is free to move.[6] The $\mathbf{B}$-field from these conductors is purely azimuthal $(\phi)$, and its radial $(r)$ dependence is determined from a straightforward un- 

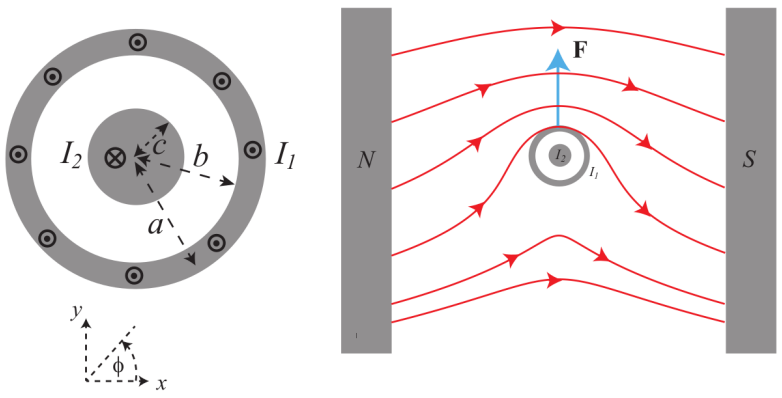

FIG. 2. (a) Concentric conductors carrying opposing currents. (b) System immersed in a uniform magnetic field, $B_{\text {magnet }}$. Parameters: $I_{2}=2 I_{1}=2 \mathrm{~A}, a=8 \mathrm{~mm}, b=6 \mathrm{~mm}, c=2 \mathrm{~mm}$, $B_{\text {magnet }}=10 \mu \mathrm{T}$.

dergraduate calculation to be

$$
\begin{array}{lrl}
B_{\phi}(r)=-\frac{\mu_{0}}{2 \pi}\left(\frac{I_{2}}{c^{2}}\right) r & r \leq c, \\
B_{\phi}(r)=-\frac{\mu_{0}}{2 \pi r} I_{2} & c<r \leq b, \\
B_{\phi}(r)=\frac{\mu_{0}}{2 \pi r}\left[\left(\frac{r^{2}-b^{2}}{a^{2}-b^{2}}\right) I_{1}-I_{2}\right] & b<r \leq a, \\
B_{\phi}(r)=\frac{\mu_{0}}{2 \pi r}\left(I_{1}-I_{2}\right) & r>a .
\end{array}
$$

For $I_{2}>I_{1}$, it is always the case that $B_{\phi}<0$, i.e. clock- wise. From cylindrical symmetry, the field from the fixed inner wire does not cause the outer cylinder to move.

Now take the structure of Fig. 2(a) and place it in the uniform field $\left(B_{\text {magnet }}\right)$ between North and South magnetic poles - see Fig. 2 (b). The figure shows the resultant field lines as calculated from Eq. (1). The field from $I_{2}$ does not impel the outer cylinder to move, which therefore moves upwards solely in response to the uniform field, contrary to the catapult picture.

In summary, we have shown that textbooks and other teaching material are 'wrong' whenever they make the explicitly causal statement that the wire moves because of the field density gradient (cf. quotes 1 and 2 above). Others (e.g. quotes 3 and 5) make a correct statement, i.e. that for the given configuration the wire moves from where the magnetic field lines are dense to where they are more rarified, but one that potentially misleads students into thinking that the picture provides the causal mechanism for why the wire moves. Quote 4 sits between the two cases, with the correct second part of the statement matching quotes 3 and 5 , but it is also conflated with a strong implication of causality from the first part ("When the two fields are combined...").

Thus, from a pedagogical viewpoint, the catapult picture ultimately fails because it encourages students, in their attempts to grasp a difficult and counterintuitive concept, into confused thinking about the forces caused by magnetic fields.

\section{ACKNOWLEDGMENTS}

Useful discussions are acknowledged with Estralita McCall, Keith Gibbs, Prof. Peter Main and Charles Tracy.
[1] K. Johnson, Physics for You (Nelson Thornes Ltd, UK, 2001).

[2] K. Johnson, S. Hewett, S. Holt, and J. Miller, Advanced Physics for You (Nelson Thornes Ltd, UK, 2000).

[3] Keith Gibbs, "schoolphysics," http://www.schoolphysics.co.uk/age16-19/ Electricity (2018), [Online; accessed 30-August-2018].
[4] "IOP Institute of Physics," http://http://www.practicalphysics.org/ catapult-magnetic-field.html (2018), [Online; accessed 30-August-2018].

[5] Nelkon and Parker, Advanced Level Physics, sixth ed. (Heinamann, 1987).

[6] To prevent Hall-type effects due to the drift of charges in each conductor, the outer cylinder can be considered to consist of a ring of insulated current-carrying wires, and the radius of the inner wire can be made sufficiently small $(c \ll a, b)$. 